\title{
Biosafety assessment and benefits for co-existence of biological contained plants- regulatory assessment in the EU-project "Transcontainer"
}

\author{
C. Koziolek \\ Federal Office for Consumer Protection and Food Safety, Berlin, Germany \\ Correspondence to: Dr. Christiane Koziolek, Federal Office for Consumer Protection and Food Safety (BVL), \\ Mauerstr. 39-42, D-10117 Berlin, Germany, Tel. +49 30444 40415, E-mail: Christine.Koziolek@bvl.bund.de
}

One goal of the EU-project TransContainer is the evaluation of environmental impact and benefits for coexistence between GM- and non-GM plants. Different containment strategies are applied to a broad spectrum of crops and perennial plants. The containment methods focus on three strategies: Chloroplast Transformation, Controllable Flowering and Controllable Fertility. Key issues are (a) safety assessment focussing on molecular characteristics, ecology of the GM species and consequences of a potential break-down of the containment system. (b) Benefit assessment of the containment system for the co-existence of GM and non-GM plants. The environmental impact assessment is performed based on the criteria provided by the EFSA Guidance document (2006) for the placing on the market of GM plants in the EU. Federal Office for Consumer Protection and Food Safety (BVL) performs a safety assessment of the general methodical characteristics of the containment systems taking into account the characteristics of the particular model plants.

Benefits of Chloroplast Transformed plants: Chloroplast transformation is a promising containment system for plant species with strict maternal plastid inheritance by avoiding the out-crossing of recombinant genes via the pollen. The targeted insertion of a GM sequence in the chloroplast genome by homologous recombination has certain advantages, e.g. avoidance of unknown functional genes by insertional inactivation. However, it has to be verified that the transgene is not inserted unintentionally in the nuclear genome since the transformation could be unspecific.

Benefits of plants with Controlled Flowering: The suppression of flowering is useful for plants that are cultivated for their vegetative parts, e.g. sugar beet or trees. For bi-annual sugar beet, the inhibition of undesired bolting and flowering will either prevent out-crossing and introgression into endogenous beet populations as well as facilitate the cultivation of beets for the farmer. In trees that are intended to be cultivated in plantations for biomass production, the suppression of flower development would offer the opportunity to cultivate GM trees with e.g. changed wood properties, avoiding any unintended hybridisation or spread of the GM seeds by wind. In plants like sugar beet, that need to flower for breeding purposes, a molecular switch will be introduced, which will initiate flowering upon an external chemical stimulus. In case of flower induction for breeding, other (physical) measures are requested to avoid unintended out-crossing and spread of hybrids.

Benefits for Co-existence: Regarding the legislation on GM plants in the EU, the minimisation of GM escape via pollen into adjacent non-GM fields or wild relatives is an important point in the improvement of co-existence measures. In chloroplast transformed plants, the spread of inserted DNA is limited only at the pollen level, whereas the (transgene) seeds produced by the mother plant could still be lost or spilled unintended during harvest and transport. Upon complete flower suppression, neither pollen nor seeds will be released, thus gaining a very high level of restriction. The containment measures will allow (i) minimising the isolation distances between GM and nonGM fields, (ii) protecting the GM cultivating farmers for liability claims from neighbours, (iii) reduce conflicts with bee keepers, and (iv) avoiding unintended mixtures with food products.

\section{Literature}

EFSA (2006) Guidance document on the scientific panel on genetically modified organisms for the risk assessment of genetically modified plants and derived food and feed. EFSA J 99:1-105. 